\title{
L'étude par modèle mathématique de l'aquifère de la craie dans la région de Moulle (Pas-de-Calais)
}

\author{
Mathematical model study of a chalk aquifer \\ near Moulle (Pas-de-Calais)
}

\author{
Gérard Dassonville \\ Société Lyonnaise des Eaux et de l'Éclairage
}

\author{
Yves Emsellem
}

Société d'études ARLAB
La S.L.E.E. a réalisé avec la collaboration de la Société ARLAB un modèle mathématique de la nappe exploitée dans la région de Moulle (Pas de Calais) pour lalimentation en eau de la région dunkerquoise et d'une vingtaine de communes du département du Pas-deCalais. Cette nappe fait l'objet d'une alimentation artificielle qui peut atteindre actuellement 8 millions de mètres-cubes par an.

L'alimentation artificielle est effectuée au moyen d'un bassin d'infiltration et utilise une eau de rivière de qualité médiocre qui fait l'objet d'un traitement complet onéreux. Le but assigné initialement à cette réalimentation était, comme c'est le cas pour la plupart des realisations de cette nature en Europe, de restaurer un champ captant devenu peu à peu surexploité par suite, non seulement de l'accroissement de nos propres pompages dans le champ captant mais aussi de tous les pompages dans le bassin versant de l'Aa dont la région de Moulle constitue une faible partie. Cette réalimentation fut mise en œuvre en 1971 en toute hâte, à la suite d'un déficit pluviométrique particulièrement sévère : l'effet conjugué d'un prélèvement à la nappe important au cours de l'année 1970 et l'absence quasi-totale de réalimentation saisonnière au cours de l'hiver 1970-71 nous ont révélé brutalement l'insuffisance des ressources pour faire face aux besoins.

L'expérience de plusieurs années d'exploitation de cette ressource nouvelle nous a conduits à concevoir la réalimentation dans une optique légèrement différente de ce qu'elle était à l'origine, et à chercher à optimiser la gestion de la réserve souterraine emmagasinée, en fonction des besoins du réseau de distribution. Une connaissance précise des mécanismes de recharge de l'aquifère et de stockage intersaisonnier constitue le seul moyen de tirer le meilleur parti du potentiel hydraulique à partir d'une modulation appropriée des réalimentations artificielles. Aussi l'objectif assigné à l'étude par modèle a-t-il été d'effectuer des prévisions sur le comportement futur de la nappe au moyen d'un outil de calcul reproduisant le plus exactement possible les écoulements souterrains.

L'étude a comporté trois phases :

- une phase d'examen et de mise en forme des données disponibles en vue de leur introduction dans le modèle,

- une phase d'étalonnage du modèle,

- une phase de prévision.

Nous allons donner ci-après quelques-uns des résultats obtenus à l'issue de cette phase de prévision.

Le programme de calcul utilisé est le programme NEWSAM et l'on a employé quatre tailles de mailles : $300,600,1200$ et $2400 \mathrm{~m}$ de côté. L'échelle de représentation adoptée est le $1 / 25000$.

\section{Hydrogéologie de la nappe de Moulle}

L'aquifère concerné est la nappe de la craie qui descend des collines d'Artois pour s'enfoncer sous les formations éocènes de la plaine des Flandres. Le champ captant de Moulle est situé en bordure de ce recouvrement de la craie, à l'Ouest de Saint-Omer. Il s'étend sur environ $10 \mathrm{~km}$ et comporte une douzaine de forages dont la profondeur est comprise entre 50 et $100 \mathrm{~m}$. On peut distinguer deux parties dans ce champ captant : - un ensemble de 6 forages concentrés à Houlle et Moulle ; ce sont les plus anciens, et la dépression de la surface de la nappe y est la plus importante. C'est dans cette zone qu'ont été creusés les bassins d'infiltration utilisés pour l'alimentation artificielle de la nappe;

- une série de captages disposés parallèlement à la limite du recouvrement de la craie par les formations tertiaires, à Eperlecques et Bayenghem-lesEperlecques.

Les limites de l'impluvium qui contribue à alimenter le champ captant de Moulle sont constituées : 


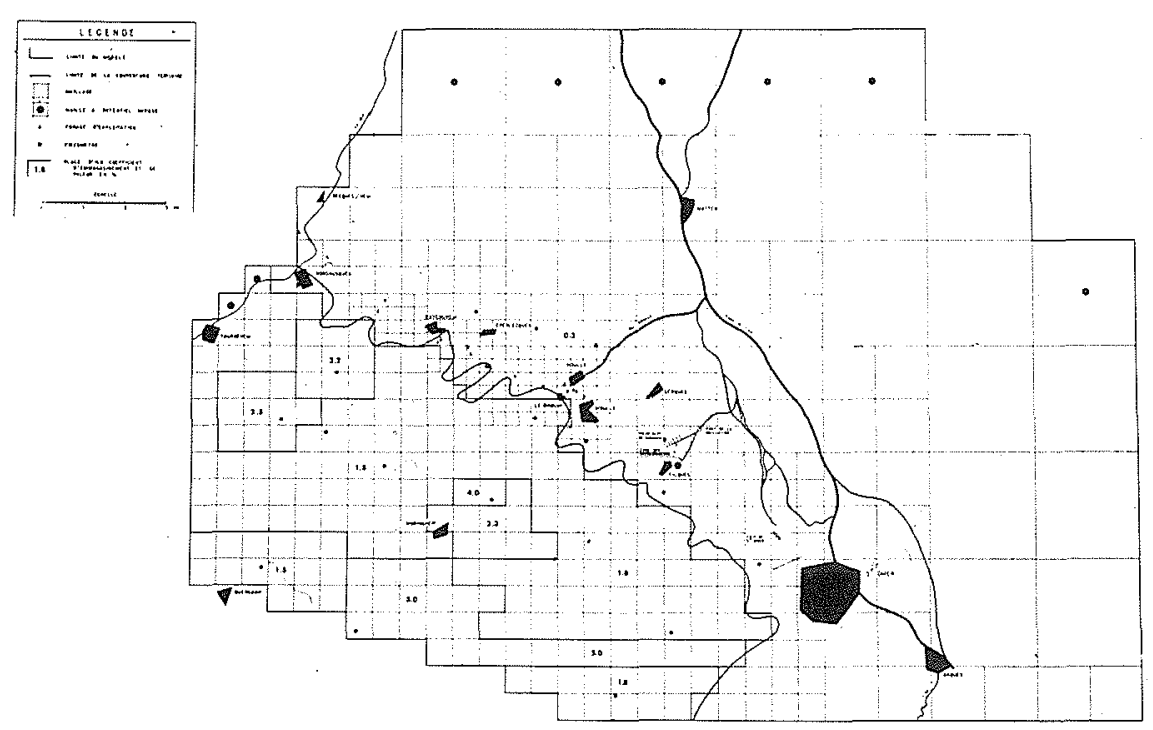

Figure 1 - Plan de position et carte piézométrique de référence

- au Sud, par la ligne de partage des eaux entre le Bléquin et l'Aa,

- au Nord, par la limite d'affleurement de la craie. Nous avons pris comme limites de la région étudiée à l'Ouest et à l'Est, les méridiens de Tournehem et de Saint-Omer.

La formation crayeuse affleure sur une surface d'environ $85 \mathrm{~km}^{2}$.

Entre ces limites Sud et Nord, la nappe est libre. Elle devient captive sous le recouvrement tertiaire, et artésienne à sa bordure ; c'est dans cette région qu'ont été creusés les premiers puits Artésiens.

Les formations tertiaires qui recouvrent la craie comprennent de bas en haut : des argiles, des sables, puis de nouveau des argiles extrêmement compactes (Argile des Flandres) et dont l'épaisseur dépasse rapidement plusieurs dizaines de mètres.

\section{Alimentation de la nappe par la pluie}

La pluviométrie varie assez sensiblement dans la région comme en témoignent les valeurs données ciaprès (lame d'eau annuelle en $\mathrm{mm}$ à Moulle) pour la dernière décennie. $\mathrm{Ce}$ sont les pluies d'hiver qui assurent l'alimentation de la nappe et celles-ci ont montré un écart de plus de un à trois entre les extrêmes de l'hiver $1970-71$ et $1974-75$.

\begin{tabular}{|l|l|l|l|l|l|l|l|l|l|l|}
\hline 1967 & 1968 & 1969 & 1970 & 1971 & 1972 & 1973 & 1974 & 1975 & 1976 & 1977 \\
\hline 597 & 667 & 567 & 497 & 407 & 506 & 521 & 922 & 875 & 570 & 765 \\
\hline
\end{tabular}

Il est évident que ces caprices du temps affectent très sensiblement et de manière variable les fluctuations du niveau de la nappe dans la région. D'après notre piézomètre de référence situé au centre de la dépression de Moulle, on peut estimer à trois à quatre mois le temps de réponse à la réalimentation saisonnière, les hautes eaux de la nappe se situant à environ six à sept mois après le début de la pluie efficace. En d'autres termes, le "transi- toire" de réalimentation du champ captant peut ètre pris égal à trois mois et la durée totale de la réalimentation à six mois.

L'absence quasi-totale de réalimentation de la nappe au cours de l'hiver 1970-71 a fait chuter de plus de 25 mètres le niveau de la nappe à Moulle en un peu plus de six mois.

La répartition saisonnière de la pluie efficace, mise en évidence par l'application de formules d'évapotranspiration potentielle mensuelle (Thornthwaite, Turc) permet d'individualiser, d'une manière générale, deux périodes distinctes durant l'année :

- une période de recharge hivernale entre octobre et mars,

- une période pratiquement sans recharge entre avril et septembre.

Cette distinction permet de classer les années selon leur pluviométrie et l'on observe une certaine répétition - au cours de la période de mesures pluviométriques à Moulle, c'est-à-dire depuis 1950- d'une succession de deux années "sèches" consécutives.

L'étalonnage du modèle a porté sur la période 1974. 1978. Les valeurs (lame d'eau en $\mathrm{mm}$ ) de la pluviométrie hivernale mesurée à Moulle et de la pluie efficace sont les suivantes :

\begin{tabular}{|c|c|c|}
\hline Hiver & Pluviométrie & Pluie efficace \\
\hline $74 / 75$ & 1031 & 530 \\
$75 / 76$ & 504 & 232 \\
$76 / 77$ & 849 & 327 \\
$77 / 78$ & 390 & 220 \\
\hline
\end{tabular}

Cette période est faite d'une succession d'années moyennement pluvieuses à sèches, après une année exceptionnellement humide. Cette forte pluviosité de l'hiver 74.75 a provoqué une remontée générale de la nappe jusqu'à saturation de l'aquifère, se traduisant par la réapparition de sources taries depuis longtemps 
le long de la ligne où la formation crayeuse s'enfonce sous la couverture tertiaire. Cette situation de "nappe pleine" constitue une excellente référence pour un calage en régime transitoire et c'est pourquoi elle a été choisie comme situation initiale.

\section{Alimentation artificielle}

Les volumes introduits dans la nappe à Moulle par l'alimentation artificielle ont été les suivants (millions de $\left.\mathrm{m}^{3}\right)$ :

\begin{tabular}{|c|c|c|c|c|c|c|}
\hline 1971 & 1972 & 1973 & 1974 & 1975 & 1976 & 1977 \\
\hline 1 & 3 & 4 & 4 & 0,5 & 5 & 4,5 \\
\hline
\end{tabular}

Plus précisément pour la période d'étalonnage du modèle, ces:débits sont les suivants : (en 1/s)

\begin{tabular}{|l|r|r|r|r|r|r|r|r|r|}
\hline & \multicolumn{2}{|c|}{1975} & \multicolumn{2}{c|}{1976} & \multicolumn{2}{c|}{1977} & \multicolumn{3}{c|}{ Moyennes } \\
\cline { 2 - 10 } & Hiver & Eté & Hiver & Eté & Hiver & Eté & Hiver & Eté & Annuelle \\
\hline Brouay & 15,7 & 4,7 & 125,9 & 192,2 & 107,7 & 151,8 & 83,1 & 116,2 & 99,7 \\
Galerie $F_{5}$ & 1,9 & 0 & 0 & 0 & 5,8 & 7,3 & 2,6 & 2,6 & 2,6 \\
\hline Débit total & 17,6 & 4,7 & 125,9 & 192,2 & 113,5 & 159,1 & 85,7 & 118,8 & 102,3 \\
\hline
\end{tabular}

\section{Prélèvements à la nappe et sorties d'eau}

Les pompages de la S.L.E.E. dans la nappe ont atteint en 1970 le cube maximum de 16,6 millions de $\mathrm{m}^{3}$ pour l'année. Au cours de la période qui suivit, ce cube annuel a été compris entre 8,5 et 17 millions de $\mathrm{m}^{3}$, en ne tenant pas compte des volumes pompés provenant de la réalimentation artificielle de la nappe.

D'autres prélèvements à la nappe par forages sont faits par la Société des Eaux de Saint-Omer, non loin des captages de la S.L.E.E.

Les valeurs des débits moyens saisonniers pompés ont été affichés dans les mailles du modèle intéressées pour la période de référence 1975-77.

Le total de ces débits pour les différentes mailles est le suivant : $(1 / \mathrm{s})$

\begin{tabular}{|c|c|c|c|c|c|}
\hline \multicolumn{2}{|c|}{1975} & \multicolumn{2}{c|}{1976} & \multicolumn{2}{c|}{1977} \\
\hline Hiver & Eté & Hiver & Eté & Hiver & Eté \\
\hline 592 & 658 & 641 & 641 & 647 & 640 \\
\hline
\end{tabular}

Les prélèvements par pompage ne constituent pas les seules sorties d'eau de la nappe de Moulle. Il existe à Tilques, en bordure de la dépression de la surface de la nappe, des cressonnières alimentées par des forages artésiens. Sur le modèle, nous avons simulé ces sorties d'eau sous la forme d'un débit d'échange entre une maille à potentiel imposé et les mailles voisines. Le trop-plein de ces cressonnières se déverse dans un réseau de petits canaux recueillant par ailleurs les eaux de ruissellement en provenance du bassin versant amont et qui convergent dans un canal principal équipé d'un déversoir. Le débit mesuré en ce point représente des valeurs par excès du débit artésien de la nappe. Nous donnons ci-dessous les valeurs de ce débit annuel (millions $\mathrm{m}^{3}$ ). Ce débit n'est connu avec précision que depuis le milieu de l'année 1971 seulement, et nous avons tenté de l'évaluer, à l'aide des mesures existantes, pour 1960 et 1969

\begin{tabular}{|c||c||c|c|c|c|c|c|c|}
\hline 1960 & 1969 & 1971 & 1972 & 1973 & 1974 & 1975 & 1976 & 1977 \\
\hline 6 & 7 & 3,5 & 4,5 & 2 & 3 & 7 & 5 & 5,5 \\
\hline
\end{tabular}

Rappelons que l'alimentation artificielle de la nappe est en service depuis 1971 et remarquons que les volumes reinjectés ont été supérieurs aux sorties d'eau des cressonnières en 1972, 73 et 74 , après l'épisode de pluviosité déficitaire.

$\mathrm{Au}$ total, et pour la période où les débits sont connus avec une certaine précision, les sorties d'eau de la nappe (millions $\mathrm{m}^{3}$ ) sont les suivantes :

\begin{tabular}{|c|c|c|c|c|c|c|c|c|}
\hline 1969 & 1970 & 1971 & 1972 & 1973 & 1974 & 1975 & 1976 & 1977 \\
\hline 24 & $>19^{*}$ & 18 & 13 & 12,5 & 16 & 26 & 20 & 21 \\
\hline
\end{tabular}

La valeur correspondant à l'année 1975 est exceptionnelle : bien que l'alimentation artificielle ait été très faible, $\left(0,5 \mathrm{Mm}^{3}\right)$ les pompages ont retrouvé une valeur du même ordre qu'en 1970 - avant réalimentation artificielle - et les sorties d'eau des cressonnières ont atteint un des maxima connus. Cette situation est la conséquence de la pluviọsité exceptionnelle de l'hiver 1974-75.

\section{Paramètres hydrauliques de l'aquifère}

La répartition des transmissivités mesurées est très inégale sur le domaine d'étude. Elle s'exprime généralement en $10^{-2} \mathrm{~m}^{2} / \mathrm{s}$ dans le champ captant pour une piézométrie normale. Après une évaluation de sa valeur dans les secteurs où celle-ci n'est pas connue, l'ajustement de la transmissivité a été fait par approximations successives à l'aide du modèle, en tenant compte de l'alimentation de la nappe par l'infiltration efficace, des conditions aux limites et de la piézométrie. La carte des transmissivités qui a pu être établie montre une large zone de transmissivité élevée $\left(2\right.$ à $\left.5.10^{-2} \mathrm{~m}^{2} / \mathrm{s}\right)$ entre Bayenghem-les-Eperlecques et Saint-Omer ; sur le bassin d'alimentation les valeurs sont plus faibles (entre 0,6 et $6.10^{-3}$ ).

Pour le coefficient d'emmagasinement, les données sont peu nombreuses; on estime à $3 \%$ sa valeur dans la partie Ouest du champ captant et nous avons considéré que cette valeur pouvait être conservée sur toute la bordure du recouvrement tertiaire, tant que l'épaisseur de celui-ci ne dépasse pas $10 \mathrm{~m}$.

Bien entendu, comme pour la transmissivité, l'ajustement des valeurs du coefficient d'emmagasinement a été fait au cours du calage du modèle. $S$ est compris entre 1,5 et $5 \%$ sur le bassin d'alimentation et égal à $0,3 \%$ en ce qui concerne l'aquifère captif.

La diffusivité de la craie apparaît donc élevée et les réactions de la nappe sont "nerveuses". Aucune des 


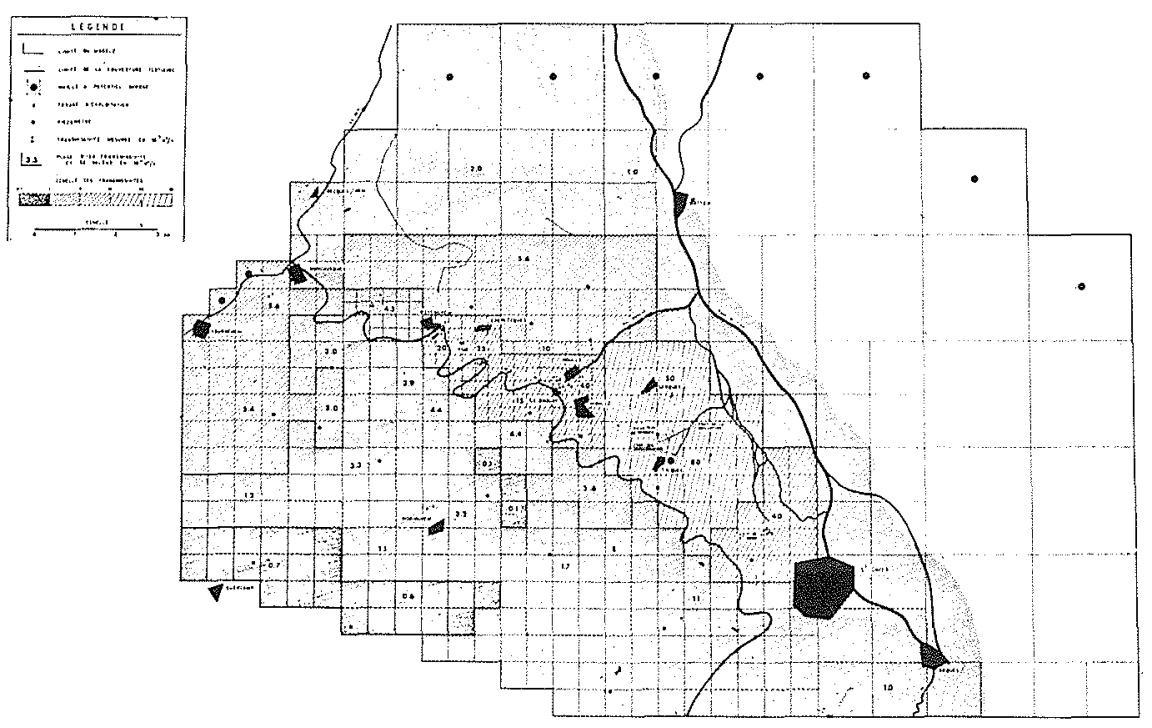

Figure 2 - Carte de transmissivité

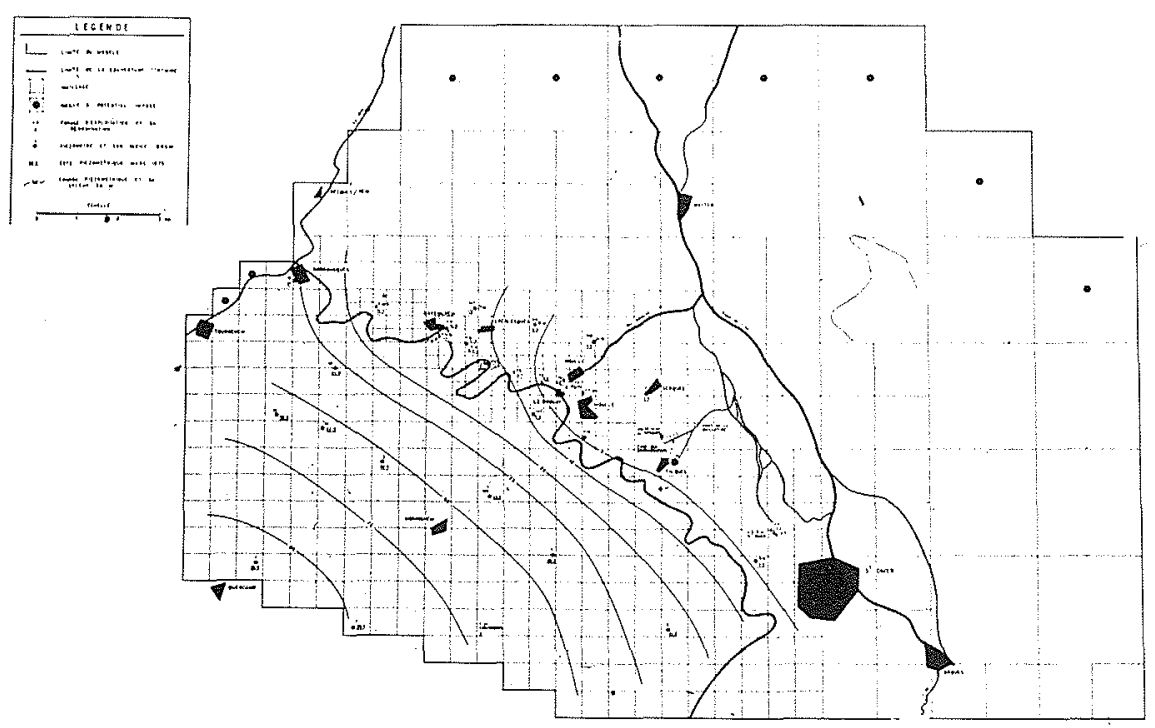

Figure 3 - Carte du coefficient d'emmagasiment

observations disponibles ne permet d'invoquer la sollicitation de l'aquifère sus-jacent à la craie (nappe des sables landéniens) par les pompages. Cette drainance, si elle existe, doit avoir un caractère très local.

\section{Piézométrie et pertes de charge}

La piézométrie de la nappe est assez bien connue, autant dans l'espace que dans le temps. Nous voudrions toutefois souligner que l'existence d'une vaste dépression autour des captages de Moulle poserait le problème de l'écoulement en provenance du Nord. On sait que la craie s'enfonce sous une couverture imperméable épaisse et qu'elle devient de moins en moins perméable à mesure que l'on s'éloigne de son affleurement. Que signifierait par conséquent un gradient d'écoulement de la nappe captive, du Nord vers le Sud, à Moulle ? Nous avons examiné plusieurs hypothèses pour tenter d'apporter une explication : a) erreur sur les niveaux piézométriques

Les piézomètres pouvant servir à représenter la dépression de Moulle dans la direction du Nord sont peu nombreux et, compte tenu de la structure géologique, on peut se demander si le niveau d'eau que l'on y mesure est bien celui de la nappe de la craie. Nous avons réalisé deux nouveaux piézomètres pour lever cette incertitude.

b) les niveaux piézométriques des forages exploités sont affectés de pertes de charge importantes.

Cette hypothèse a été vérifiée par l'étude des courbes de remontée de pression et il nous est apparu que les pertes de charge dans les tubes de forage représentent un pourcentage élevé du rabattement en pompage. Il en résulte par conséquent que la dépression réelle de la nappe à Houlle et à Moulle est moindre qu'il n'y paraît au premier coup d'œil.

c) reste le problème de la réalimentation induite à partir des eaux de surface. Le calage du modèle a mis en 
évidence une dérivation au profit de la vallée de la Hem dans la région d'Eperlecques.

\section{Etalonnage du modèle}

Après une série d'essais en régime permanent en vue de s'assurer de la cohérence des données introduites dans le programme et de fournir un état piézométrique initial pour le transitoire, l'étalonnage du modèle a été effectué par la suite en régime transitoire d'écoulement, c'est-à-dire qu'il a consisté à reproduire au moyen du calcul la piézométrie de la nappe mesurée dans le temps en différents points de surveillance piézométrique du domaine d'étude.

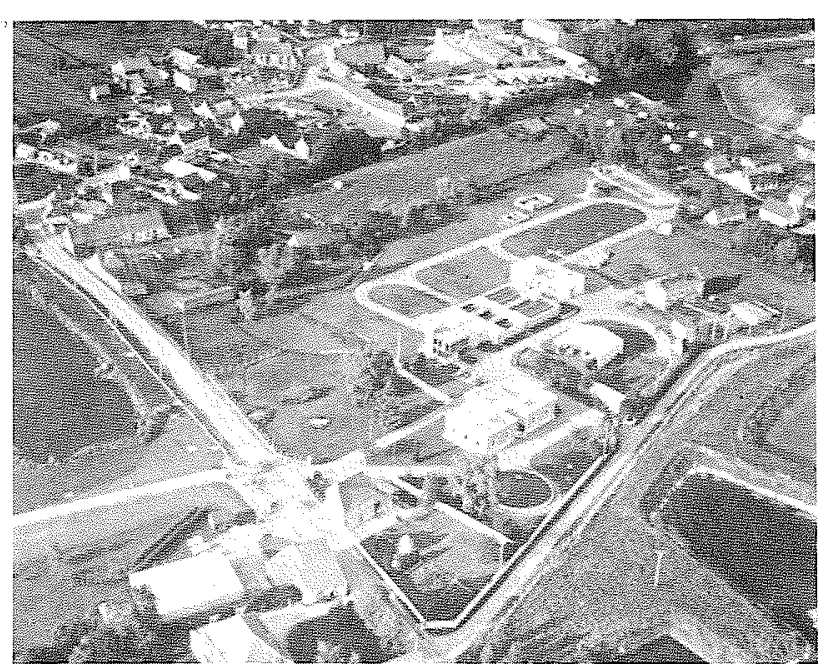

Figure 4 Vue générale des installations

(Phot'I - cliché aéroport Lille Lesquin)

Le procédé d'étalonnage a donc consisté à s'appuyer sur un état initial relativement stable, c'est-à-dire qui soit le plus possible indépendant de l'historique de ce qui précède. Cette situation s'est présentée à la suite de l'hiver exceptionnel 1974-75 qui a provoqué une remontée spectaculaire de la nappe jusqu'à "remplissage" complet de l'aquifère à la fin de la saison. Cette situation a donc été choisie comme état de référence ; par la suite, on s'est attaché à reproduire à l'aide du modèle les variations de la piézométrie observée au cours des saisons successives : été 75 , hiver 75-76, été 76 , hiver 76-77, en ajustant, par approximations successives les différents paramètres suivants::

- l'infiltration efficace, c'est-à-dire un pourcentage de la pluie efficace, dont la valeur nous est apparue comme devant être égale à $90 \%$ de la pluie efficace calculée à l'aide de la formule de Thornthwaite ;

- la transmissivité et le coefficient d'emmagasinement.

Un étalonnage satisfaisant du modèle a été obtenu après une soixantaine de tests en régime transitoire qui ont précisé les débits aux limites, les débits du drainage insoupçonné par la Hem et les débits artésiens dans la région de Tilques.
Phase de prévision.

La prévision des piézométries pour l'été 77 et l'hiver 77-78 a tout d'abord été faite, afin notamment de vérifier au bilan la diminution de l'infiltration efficace par rapport à l'hiver $76-77(10501 / \mathrm{s}$ au. lieu de 1560$)$ et son effet sur la chute du débit artésien à Tilques. A partir de là, et pour en faire la vérification en fonction des données réelles à la fin de l'année 1978, plusieurs prévisions ont été faites pour l'été 1978.

\section{Hypothèse $n^{\circ} I$ : réalimentation saisonnière normale de $10000 \mathrm{~m}^{3} / \mathrm{j}$}

Les prélèvements saisonniers ont èté affichés sur le modèle pour un débit égal à la moyènne des trois dernières années (cf. plus haut). De même, pour la réalimentation artificielle, on a pris le débit moyen saisonnier de $120 \mathrm{l} / \mathrm{s}$ (soit $10000 \mathrm{~m}^{3} / \mathrm{j}$ en chiffre rond).

La carte piézométrique prévisionnelle pour la fin de l'été 78 montre que la nappe s'abaisse de manière importante à la fois dans le bassin d'alimentation et dans le champ captant de Moulle malgré la réalimentation. Elle reste en position relativement haute cependant dans la région d'Eperlecques, d'où le fort drainage enregistré pour la Hem. Une telle situation ne permettrait pas le maintien d'un débit artésien dans les cressonnières de Tilques.

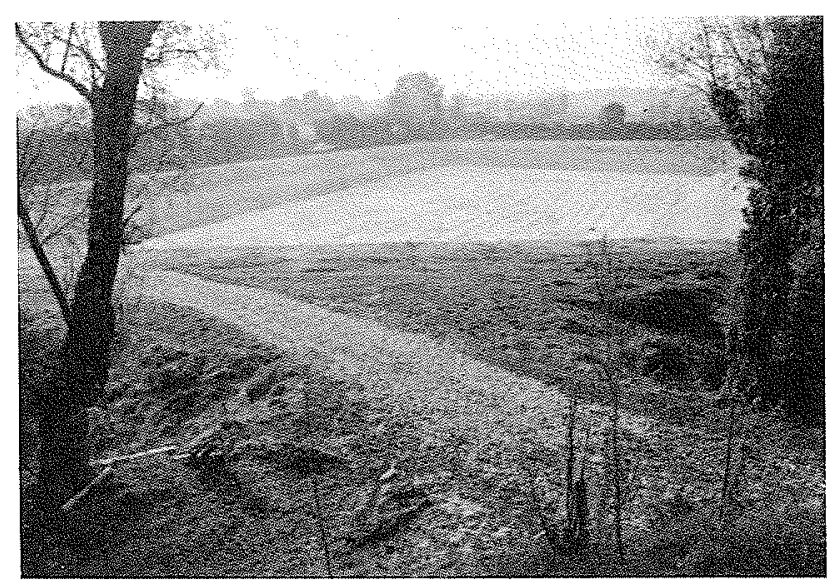

Figure 5 Bassin d'infiltration

\section{Hypothèse $n^{\circ} 2$ : réalimentation poussée au débit de $20000 \mathrm{~m}^{3} / \mathrm{j}$.}

Le résultat de l'injection supplémentaire de $110 \mathrm{1} / \mathrm{s}$ est spectaculaire et le bilan des flux indique que le débit artésien à Tilques passe de 0 à $30 \mathrm{l} / \mathrm{s}$.

A l'issue de ces deux scénarios, les conclusions que l'on pouvait tirer pour l'exploitation étaient les suivantes:

augmenter dans toute la mesure du possible les prélèvements dans la partie Ouest du champ captant et choisir un débit de réalimentation intermédiaire entre 10000 et $20000 \mathrm{~m}^{3} /$ jour.

Plutôt que nous en tenir là, nous avons préféré tester un autre scénario. 


\section{Hypothèse $n^{\circ} 3:$ modulation mensuelle de la réalimen- tation}

Une répartition mensuelle de la réalimentation a été étudiée à l'aide du modèle, de manière à accroître l'efficacité de cette réalimentation tout en assurant un débit artésien minimum à Tilques. Les débits $(1 / \mathrm{s})$ de la réalimentation mensuelle à mettre en œuvre que nous donne le calcul par le modèle sont les suivants :

\begin{tabular}{|l|r|r|r|r|r|r|r|}
\hline & Avril & Mai & Juin & Juillet & Aout & Septembre & Movenne \\
\hline $\mathrm{e} / \mathrm{s}$ & 96 & 148 & 187 & 206 & 213 & 240 & 182 \\
\hline $\mathrm{m}^{3} / \mathrm{i}$ & 8300 & 12800 & 16200 & 17800 & 18400 & 20700 & 15700 \\
\hline
\end{tabular}

Une telle répartition des débits permet une substancielle économie de l'eau de réalimentation, tout en conservant une piézométrie raisonnablement haute et un débit artésien à Tilques d'une dizaine de $1 / \mathrm{s}$.

\section{Prévisions sur le comportement futur de la nappe}

Ces prévisions concernent l'hiver $78-79$ et l'été 79 , en prenant en compte la recharge de l'aquifère par la pluie au cours de l'hiver $78-79$ selon les 3 types d'années :

- pluvieuses : plus de $500 \mathrm{~mm}$ de pluie hivernale, - moyenne : $300 \mathrm{~mm}$ de pluie hivernale,

- sèche : $200 \mathrm{~mm}$ de pluie hivernale.

\section{Année pluvieuse 78-79}

Deux scénarios en ce qui concerne la réalimentation artificielle :

a) scénario $n^{\circ} 1$ :

- mêmes pompages que précédemment ;

- réalimentation artificielle pendant l'hiver $78-79$ nulle ;

- réalimentation durant l'été 79 à $10000 \mathrm{~m}^{3} / \mathrm{j}$

On observe au cours de toute la période comprise entre l'été 78 et l'été 79 un abaissement général de la nappe particulièrement sensible dans le secteur de Moulle et l'annulation du débit artésien de Tilques à la fin de l'été 79 .

b) scénario $\mathrm{n}^{\circ} 2$ :

- réalimentation artificielle pendant l'hiver 78-79 portée à $7500 \mathrm{~m}^{3} / \mathrm{j}$;

- réalimentation d'été $20000 \mathrm{~m}^{3} / \mathrm{j}$.

La situation est cette fois "confortable" et une situation "acceptable" pourrait être obtenue avec une réalimentation d'été moins forte.

En conclusion, le dispositif actuel de réalimentation permettra de faire face à la situation. Nous avons alors testé le cas de l'année moyenne.

\section{Année moyenne 78.79}

Trois hypothèses ont été testées : a) scénario $n^{\circ} 1$ :

- pompage S.L.E.E. et St Omer égaux à la moyenne des 3 années 75-76-77;

- réalimentation artificielle moyenne en hiver 13600 $\mathrm{m}^{3} / \mathrm{j}$.

- réalimentation artificielle forte en été $20000 \mathrm{~m}^{3} / \mathrm{j}$.

La situation piézométrique est normale mais le débit artésien à Tilques tend vers 0 en fin de saison.

b) scénario $n^{\circ} 2$ :

- réalimentation artificielle plus forte en hiver 15500 $\mathrm{m}^{3} / \mathrm{j}$.

- réalimentation artificielle maximale en été 20000 $\mathrm{m}^{3} / \mathrm{j}$.

- prélèvement S.L.E.E. identique en volume mais nappe maintenue en position haute dans le secteur d'Eperlecques en hiver pour constituer une réserve reprise en été.

La concentration des prélèvements sur Moulle ne permet pas de maintenir le débit artésien en hiver à Tilques.

Par contre, l'allègement des pompages sur Moulle le rétablit en été.

Il apparaît qu'il est préférable d'exploiter en hiver les deux secteurs de façon homogène et de renforcer les prélèvements à Eperlecques en été.

c) scénario $n^{\circ} 3$ :

- mêmes hypothèses que pour le scénario $n^{\circ} 1$;

- débit supplémentaire de $10 \mathrm{l} / \mathrm{s}$ à l'Est des pompages de St Omer.

Ce débit réduit le débit artésien à Tilques en hiver et l'annule en été.

\section{Année sèche 78-79}

Les résultats qui précèdent ont mis en évidence une certaine difficulté à maintenir un débit artésien à Tilques au cours et à la fin de l'été 79 , compte-tenu de l'historique de l'infiltration efficace de la pluie et des modalités d'exploitation de la nappe.

Dans le cas de l'année sèche (qui correspond à une infiltration efficace de $180 \mathrm{l} / \mathrm{s}$ durant l'hiver 78-79) cette situation s'aggraverait considérablement malgré un régime renforcé de réalimentation artificielle.

Un premier scénario a confirmé que, malgré une réalimentation de $20000 \mathrm{~m}^{3} /$ jour maintenue en permanence, le débit artésien à Tilques s'annulait pendant l'été 79 et que la situation piézométrique devenait préoccupante.

On imagine par conséquent facilement les effets d'une deuxième année sèche consécutive.

Dans un deuxième scénario, nous avons cherché à déterminer un programme de réalimentation permettant de faire face à la situation critique qui se poserait dans le cas d'un hiver 78-79 sec. Ce programme a été défini en recherchant mois par mois quel serait le débit de réalimentation nécessaire pour assurer la pérennité des débits artésiens à Tilques jusqu'à la fin de l'été 1979.

Les résultats trouvés ont été les suivants : 


\begin{tabular}{|c|c|c|c|c|c|c|c|}
\hline \multicolumn{8}{|l|}{ Hiver $78-79$} \\
\hline $\begin{array}{c}\text { Octobre } \\
78\end{array}$ & $\begin{array}{c}\text { Novembre } \\
78\end{array}$ & $\begin{array}{c}\text { Décembre } \\
78\end{array}$ & $\begin{array}{c}\text { Janvier } \\
79\end{array}$ & $\begin{array}{c}\text { Février } \\
79\end{array}$ & $\begin{array}{c}\text { Mars } \\
79\end{array}$ & $\begin{array}{l}\text { Movenne } \\
\text { saisonnière }\end{array}$ & Total \\
\hline $\begin{array}{c}190 \mathrm{l} / \mathrm{s} \\
16400 \mathrm{~m}^{3} / \mathrm{j} \\
492000 \mathrm{~m}^{3}\end{array}$ & $\begin{array}{c}220 \mathrm{l} / \mathrm{s} \\
19000 \mathrm{~m}^{3} / \mathrm{j} \\
570000 \mathrm{~m}^{3}\end{array}$ & $\begin{array}{c}238 \mathrm{l} / \mathrm{s} \\
20000 \mathrm{~m}^{3} / \mathrm{s} \\
615000 \mathrm{~m}^{3}\end{array}$ & $\begin{array}{c}232 \mathrm{l} / \mathrm{s} \\
20000 \mathrm{~m}^{3} / \mathrm{s} \\
600000 \mathrm{~m}^{3}\end{array}$ & $\begin{array}{c}233 \mathrm{l} / \mathrm{s} \\
20100 \mathrm{~m}^{3} / \mathrm{j} \\
603000 \mathrm{~m}^{3}\end{array}$ & $\begin{array}{c}233 \mathrm{l} / \mathrm{s} \\
20100 \mathrm{~m}^{3} / \mathrm{j} \\
603000 \mathrm{~m}^{3}\end{array}$ & $\begin{array}{c}224 \mathrm{l} / \mathrm{s} \\
19350 \mathrm{~m}^{3} / \mathrm{j} \\
580500 \mathrm{~m}^{3}\end{array}$ & $\begin{array}{c}- \\
- \\
3483000 \mathrm{~m}^{3}\end{array}$ \\
\hline
\end{tabular}

\begin{tabular}{|c|c|c|c|c|c|c|c|}
\hline \multicolumn{8}{|l|}{ Eté 79} \\
\hline $\begin{array}{c}\text { Auril } \\
79\end{array}$ & $\begin{array}{l}\text { Mai } \\
79\end{array}$ & $\begin{array}{l}\text { Juin } \\
79\end{array}$ & $\begin{array}{c}\text { Juillet } \\
79\end{array}$ & $\begin{array}{c}\text { Août } \\
79\end{array}$ & $\begin{array}{c}\text { Septembre } \\
79\end{array}$ & $\begin{array}{l}\text { Moyenne } \\
\text { saisonnière }\end{array}$ & Total \\
\hline $\begin{array}{c}261 \mathrm{l} / \mathrm{s} \\
22550 \mathrm{~m}^{3} / \mathrm{j} \\
676500 \mathrm{~m}^{3}\end{array}$ & $\begin{array}{c}282 \mathrm{l} / \mathrm{s} \\
24400 \mathrm{~m}^{3} / \mathrm{j} \\
732000 \mathrm{~m}^{3}\end{array}$ & $\begin{array}{c}300 \mathrm{l} / \mathrm{s} \\
25900 \mathrm{~m}^{3} / \mathrm{j} \\
770000 \mathrm{~m}^{3}\end{array}$ & $\begin{array}{c}304 \mathrm{l} / \mathrm{s} \\
26300 \mathrm{~m}^{3} / \mathrm{j} \\
789000 \mathrm{~m}^{3}\end{array}$ & $\begin{array}{c}308 \mathrm{l} / \mathrm{s} \\
26600 \mathrm{~m}^{3} / \mathrm{j} \\
798000 \mathrm{~m}^{3}\end{array}$ & $\begin{array}{c}328 \mathrm{l} / \mathrm{s} \\
28300 \mathrm{~m}^{3} / \mathrm{j} \\
849000 \mathrm{~m}^{3}\end{array}$ & $\begin{array}{c}297 \mathrm{l} / \mathrm{s} \\
25700 \mathrm{~m}^{3} / \mathrm{j} \\
770000 \mathrm{~m}^{3}\end{array}$ & $\begin{array}{c}- \\
- \\
4621500 \mathrm{~m}^{3}\end{array}$ \\
\hline
\end{tabular}

\section{Conclusion}

Pour l'exploitation de la nappe cette étude est intéressante à trois points de vue :

a) Elle a confirmé des idées que nous avions acquises avec l'expérience :

- le volume disponible en année de pluviométrie moyenne est de 12 millions de $\mathrm{m}^{3}$;

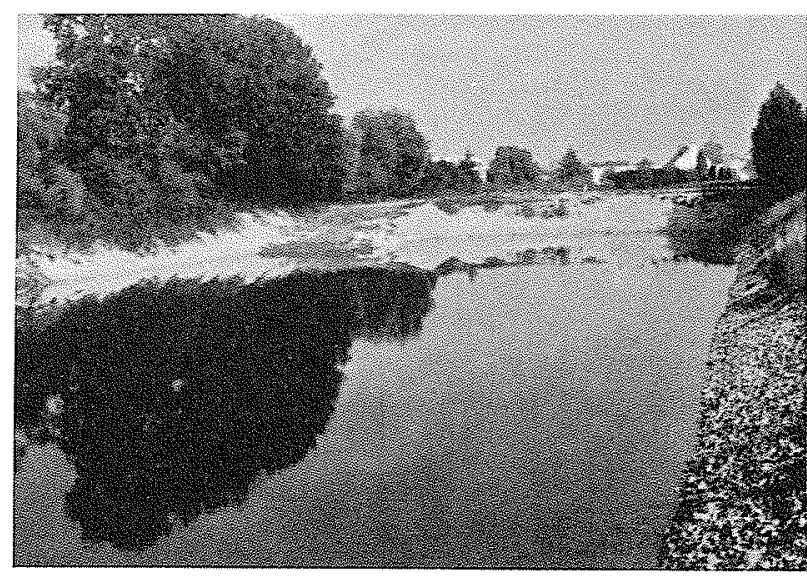

Figure 6 - Bassin d'infiltration.
- les installations actuelles ont une capacité insuffisante en cas d'hiver sec. Ceci confirme le bien fondé de la décision prise par ailleurs d'extension de la capacité de réalimentation.

b) Le modèle modifie notre schéma de pensée qui visait à équilibrer un bilan annuel, car il nous amène à intégrer :

- les débits de stockage et de destockage dans la nappe en tenant compte des variations de la piézométrie dans le bassin;

- l'intérêt de moduler mensuellement le programme de réalimentation.

c) Le modèle sera utilisé par la suite comme modèle de gestion. Nous envisageons un passage en machine tous les ans au mois d'avril. Après intégration de la pluviométrie, des pompages et de la réalimentation de l'année écoulée, le modèle définira, compte-tenu des prévisions de pompage :

- le programme de réalimentation de l'été suivant ; - des prévisions pour l'année suivante en fonction de trois hypothèses sur la pluviométrie de l'hiver : pluvieux, moyen ou sec.

Le modèle devient ainsi un véritable outil de gestion de la nappe en nous permettant une exploitation plus sûre et plus économique de celle-ci. 